\title{
Corn-derived carbohydrate inositol hexaphosphate inhibits Barrett's adenocarcinoma growth by pro-apoptotic mechanisms
}

\author{
DAVID W. McFADDEN ${ }^{1}$, DALE R. RIGGS, BARBARA J. JACKSON and CYNTHIA CUNNINGHAM \\ Department of Surgery, Robert C. Byrd Health Science Center, West Virginia University, Morgantown, WV 26506, USA
}

Received August 31, 2007; Accepted October 19, 2007

\begin{abstract}
Inositol hexaphosphate (IP6) is a naturally occurring polyphosphorylated carbohydrate that is found in food sources high in fiber content. IP6 has been reported to have significant inhibitory effects against a variety of primary tumors. We hypothesized that IP6 would inhibit the cell growth rate of Barrett's adenocarcinoma in vitro. Two Barrett'sassociated adenocarcinoma cell lines, SEG-1 and BIC-1, were treated with IP6 at $0.5,1.0$ and $5.0 \mathrm{mM}$ concentrations. Cell viability was measured by MTT assay. Apoptosis and necrosis were evaluated by the Annexin V FITC assay. Reductions $(\mathrm{P}<0.001)$ in cellular proliferation were observed in both cell lines. IP6 decreased late apoptosis and necrosis in BIC cells, whereas in SEG-1 cells, early apoptosis, late apoptosis and necrosis were all increased by IP6. IP6 decreases cellular growth by pro-apoptotic mechanisms. Our findings suggest that IP6 has the potential to become an effective adjunct for Barrett's adenocarcinoma. Further studies are needed to evaluate safety and clinical utility of this agent in patients with Barrett's adenocarcinoma.
\end{abstract}

\section{Introduction}

Gastroesophageal reflux disease and Barrett's esophagus are considered to be precursors for the development of esophageal adenocarcinoma. Barrett's, or esophageal adenocarcinoma, still remains one of the deadliest forms of gastrointestinal cancer. The mortality rate is greater than $90 \%$ in patients with this disease (1). Most patients are diagnosed during the advanced stages and survive less than a year (2). Treatments for this disease, such as surgical resection, chemotherapy, and radiotherapy, may improve survival, but exploration of other alternative and safer treatment modalities is warranted (2). Chemopreventive agents, such as nonsteroidal anti-inflammatory drugs, cyclooxygenase-2

Correspondence to: ${ }^{1}$ Present address: Professor David W. McFadden, Department of Surgery, University of Vermont College of Medicine, Fletcher House 301, 111 Colchester Avenue, Burlington, VT 05401, USA

E-mail: david.mcfadden@vtmednet.org

Key words: inositol hexaphosphate, Barrett's adenocarcinoma, cellular proliferation, apoptosis inhibitors, dietary supplements and N-3 ( $\omega-3)$ fatty acids are under investigation (2).

Esophageal adenocarcinoma usually arises from metaplastic columnar esophageal epithelium, with all of the responsible factors remaining unexplained. Increased proliferation and reduced apoptosis of the Barrett's epithelium are involved in the carcinogenesis and presumably allow accumulation and propagation of genetic abnormalities that lead to malignant transformation. Increased proliferation in Barrett's esophagus is associated with an increased risk of progression to carcinoma (3). Therefore, it will be important to establish the factors that impel proliferation or reduce apoptosis.

Inositol hexaphosphate (IP6), or phytic acid, is a naturally occurring polyphosphorylated carbohydrate that is found in foods high in fiber content such as legumes and cereals (4). It is quickly absorbed in the gastrointestinal tract (5). IP6 is also present in almost all mammalian cells, where it regulates cell function, proliferation and differentiation (5). IP6, a natural antioxidant (6), has been shown to express significant inhibitory effects against a variety of primary tumors such as breast $(7,8)$, colon (9), liver (10), prostate (11-13) pancreas (14) and melanoma (15). The effects of IP6 have not been evaluated in Barrett's adenocarcinoma; therefore we hypothesized that IP6 would significantly inhibit the cell growth rate of Barrett's adenocarcinoma in vitro.

\section{Materials and methods}

Inositol hexaphosphate. IP6 (dodecasodium salt) derived from corn (Sigma Catalog \#P8810) was purchased from Sigma Chemical Co. (St. Louis, MO). IP6 was solubilized in tissue culture media to the desired and physiologic concentrations of $0.5,1.0$ and $5.0 \mathrm{mM} /$ well.

Cell culture and reagents. Human esophageal adenocarcinoma cells (BIC-1 and SEG-1) were derived from Barrett's-associated adenocarcinomas of the distal esophagus and generously provided as a gift by David G. Beer, University of Michigan (16). The cell lines were maintained as monolayers in DMEM media with L-glutamine (Invitrogen, Carlsbad, CA), supplemented with 10\% FBS (ATCC, Manassas, VA) and penicillin/ streptomycin (Biowhittaker Inc., Walkersville, MD), and maintained in humidified air at $5 \% \mathrm{CO}_{2}$ in a $37^{\circ} \mathrm{C}$ environment. Cells were plated in sterile 96 -well microtiter plates at $1 \times 10^{5}$ cells $/ \mathrm{ml}$ and incubated for 24,48 and $72 \mathrm{~h}$ with the 
above treatments. An equal volume of vehicle was added representing the controls.

MTT assay. The MTT colorimetric assay was performed to detect tumor cell viability after 24,48 and $72 \mathrm{~h}$ of incubation. MTT, a tetrazolium dye [3-(4,5-dimethylthiazol-2-yl)-2,5diphenyltetrazolium bromide; thiazolyl blue, Sigma] was added to the each well as described previously (17). Plates were incubated in the presence of MTT dye for $4 \mathrm{~h}$. Mitochondrial dehydrogenase activity reduced the yellow MTT dye to a purple formazan, which was then solubilized with acidified isopropanol and absorbance was read at $570 \mathrm{~nm}$ on an ELx800 microtiter plate reader (Biotek Instruments Inc., Winooski, VT).

Measurement and detection of apoptosis. Annexin V-FITC Apoptosis Detection Kit (Biovision Inc., Mountain View, CA) was used to detect necrotic, early and late apoptotic activity after $18 \mathrm{~h}$ of incubation with $5 \mathrm{mM}$ IP6. After treatment as outlined above, cells $\left(1 \times 10^{6}\right)$ were collected and resuspended in binding buffer, and Annexin V-FITC and propidium iodide (PI) were added to each sample and incubated in the dark for 5 min. Annexin V-FITC binding was determined by flow cytometry $(E x=488 \mathrm{~nm} ; E m=530 \mathrm{~nm})$ using FITC signal detector (FL1) and PI staining by the phycoerythrin emission signal detector (FL2).

Statistical analysis. Determination of statistical significance was performed by analysis of variance (18). Post hoc comparison of individual concentration means with the control was completed using the Tukey-Kramer Multiple Comparison test (19). The data are reported means and standard deviations.

\section{Results}

Barrett's adenocarcinoma cell line (BIC). As shown in Fig. 1, cellular proliferation was significantly reduced in the BIC cell line by the addition of IP6 at all time-points tested. When the BIC cells were treated with $0.5 \mathrm{mM} /$ well of IP6, significant reductions in cellular proliferation were observed at $24 \mathrm{~h}(33.3 \pm 12.6 \%), 48 \mathrm{~h}(51.3 \pm 5.1 \%)$ and $72 \mathrm{~h}(48.9 \pm 3.6 \%)$ when compared to control $(\mathrm{P}<0.001)$. However, with the addition of $1.0 \mathrm{mM} /$ well IP6, more profound reductions in cellular proliferation were observed at all time-points tested

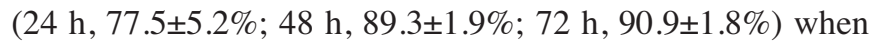
compared to control $(\mathrm{P}<0.001)$. IP6 $(5.0 \mathrm{mM} /$ well $)$ also reduced cellular proliferation at 24,48 , and $72 \mathrm{~h}(63.2 \pm 3.7$, $75.5 \pm 3.2$ and $81.5 \pm 2.3 \%$, respectively) when compared to control $(\mathrm{P}<0.001)$.

Barrett's adenocarcinoma cell line (SEG-1). When the SEG-1 cells were treated with $0.5 \mathrm{mM} /$ well IP6, cellular proliferation was reduced at $48 \mathrm{~h}(25.4 \pm 6.6 \%)$ and $72 \mathrm{~h}$ $(13.1 \pm 8.5 \%)$ when compared to control. A brief increase in SEG-1 proliferation was noted at the lowest dose $(0.5 \mathrm{mM})$ and at the earliest time-point but quickly reversed. Marked reductions in cellular proliferation were seen at the $1.0 \mathrm{mM} /$ well IP6 dose at all time-points tested $(24 \mathrm{~h}, 63.5 \pm 4.2 \%$; $48 \mathrm{~h}, 87.8 \pm 3.2 \% ; 72 \mathrm{~h}, 88.7 \pm 3.1 \%)(\mathrm{P}<0.001)$. Antiproliferative effects were also observed by the addition of

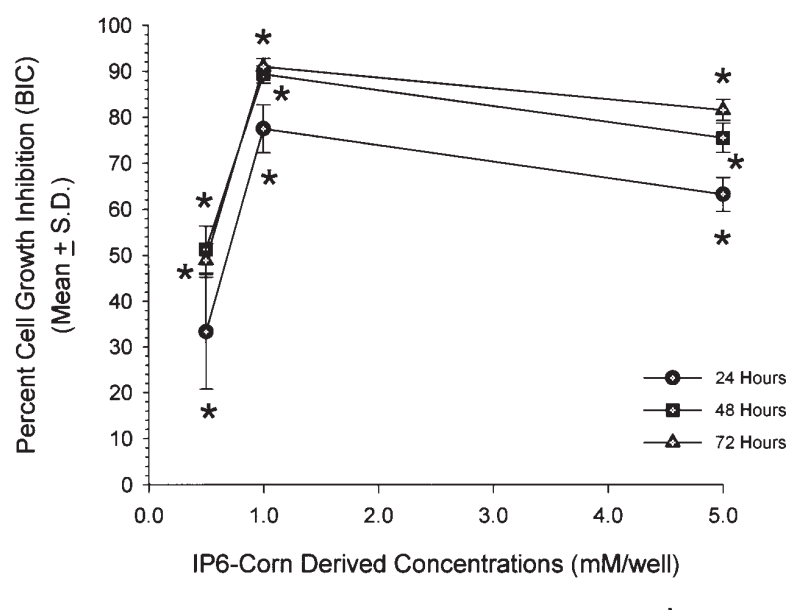

Figure 1. Cellular growth inhibition of corn-derived IP6 in the Barrett's adenocarcinoma cell line (BIC). Graph depicts the time course and dosedependence of corn-derived IP6. Data shown are means \pm standard deviations.

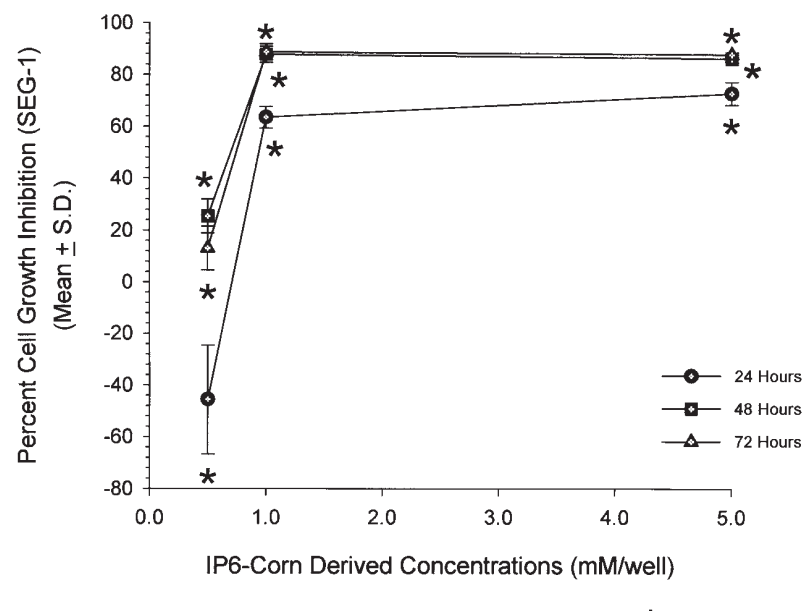

$*=P<0.001$

Figure 2. Corn-derived IP6 inhibits cellular growth in vitro in the Barrett's adenocarcinoma cell line (SEG-1) at all time-points with the exception of $0.5 \mathrm{mM}$ at $24 \mathrm{~h}$ which caused a significant proliferative effect. Graph depicts the time course and dose-dependence of corn-derived IP6. Data shown are means \pm standard deviations.

$5.0 \mathrm{mM} /$ well IP6 at 24, 48 and $72 \mathrm{~h}(72.6 \pm 4.4,86.1 \pm 1.5$ and $87.6 \pm 1.0 \%$, respectively) when compared to control $(\mathrm{P}<0.001)$, as shown in Fig. 2.

\section{Apoptosis}

Barrett's adenocarcinoma cell line (BIC). As seen in Fig. 3, late apoptosis was reduced by $5.0 \mathrm{mM}$ IP6 $(2.03 \pm 0.15 \%)$ when compared to the control $(2.93 \pm 0.42 \%, \mathrm{P}=0.025)$ in the BIC cell line. Necrotic activity was also significantly reduced when the BIC cells were treated at this dose $[(0.64 \pm 0.09 \%)$ vs. $(1.05 \pm 0.17 \%), \mathrm{P}=0.021]$.

Barrett's adenocarcinoma cell line (SEG-1). In the SEG-1 cell line, increases in both early $(2.9 \pm 1.18 \%, \mathrm{P}=0.038)$ and late $(11.9 \pm 0.71 \%, \mathrm{P}=0.002)$ apoptotic activity was observed with 5.0 mM IP6 when compared to their respective controls 


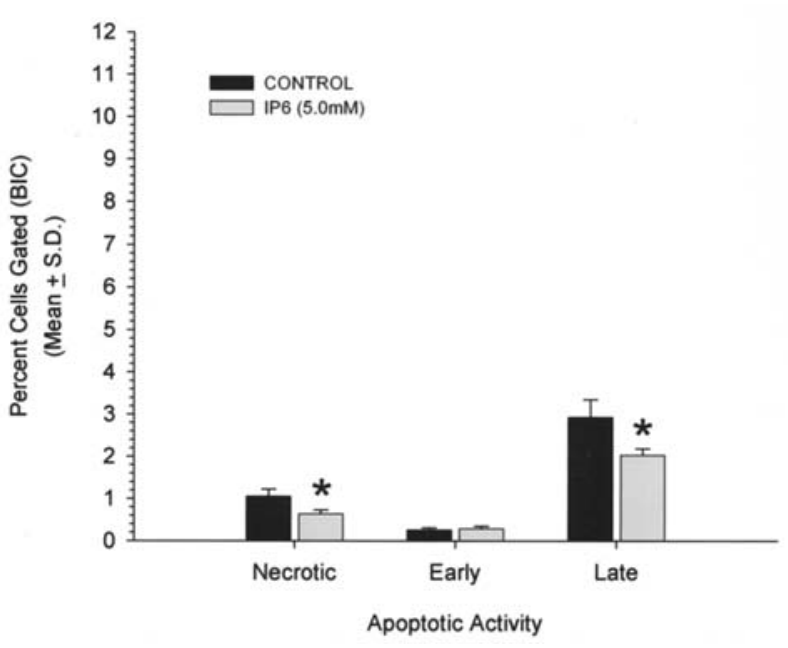

Figure 3. Late apoptotic activity and necrosis, as determined by the Annexin V assay, were significantly reduced when the BIC cell line was treated with $5.0 \mathrm{mM}$ corn-derived IP6. No significant changes were observed in early apoptosis. Graph summarizes apoptotic activity in the presence of cornderived IP6. Data shown are means \pm standard deviations.

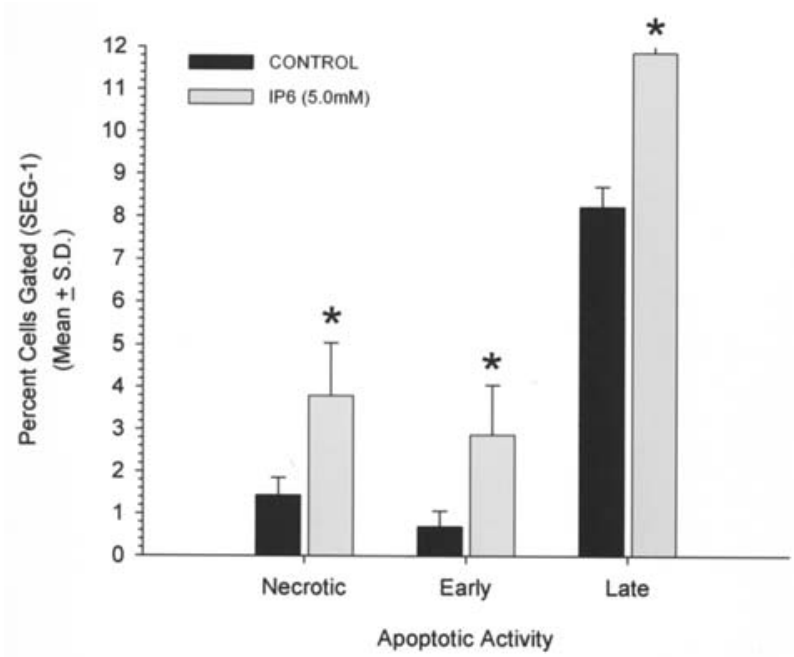

Figure 4. Apoptotic activity, determined by the Annexin V assay, induced early and late apoptosis as well as necrosis in the SEG-1 cell line when treated with $5.0 \mathrm{mM}$ corn-derived IP6. Graph summarizes apoptotic activity in the presence of corn-derived IP6. Data shown are means \pm standard deviations.

$(0.69 \pm 0.38$ and $8.2 \pm 0.47 \%)$. Necrotic activity was also significantly increased with $5.0 \mathrm{mM}$ IP6 $(3.8 \pm 1.3 \%, \mathrm{P}=0.036)$ when

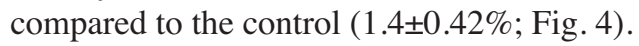

\section{Discussion}

Barrett's esophagus, first described in 1950, occurs when the distal esophagus becomes partially lined with columnar epithelium of the intestinal metaplasia subtype. The associated cancer risk of Barrett's esophagus is widely debated; however, investigators agree that a 20 - to 40 -fold increase in cancer risk exists $(20,21)$. The incidence of Barrett's associated adenocarcinoma of the esophagus continues to increase in the United States, with an estimated 15,550 new cases of esophageal cancer in 2007, with 13,940 of these cases resulting in death (22). Devesa et al evaluated incidence trends in the United States and noted a $350 \%$ increase in the incidence of esophageal adenocarcinoma between 1976 and 1994 (23). In the last seven years the relative mortality rate attributed to esophageal adenocarcinoma has declined from $98.4 \%$ in the year 2000 to an estimated $89.6 \%$ in 2007 (22). This decline can be attributed to advances in surgical techniques and treatment modalities. However, both surgical and adjuvant therapies are of limited efficacy in advanced disease or in debilitated patients. Prevention, either by medical, dietary or surgical therapies, remains highly debated. Progress in this area, however, has been limited by the development of research models to study the disease. The recent development of an in vitro model of Barrett's adenocarcinoma has allowed basic research into its growth and regulation (24).

IP6 is a naturally occurring polyphosphorylated carbohydrate found in grains, cereals, nuts, and other food sources high in fiber content. IP6 is a natural antioxidant and has been reported to have significant inhibitory effects against a variety of primary tumors. Additionally, Deliliers et al reported that IP6 is neither cytotoxic nor cytostatic against normal cells; rather its effects are cancer cell specific. In a dose titration of IP6's cytotoxic effects on leukemic progenitors cells, their group reported that no effect of IP6 was observed on normal progenitor $\mathrm{CD} 34^{+}$cells derived from bone marrow (25). No dominant theory exists for the anticancer activity of inositol compounds. Inositol phosphates, which are present in almost all mammalian cells, play an important physiologic role in regulating cell function, proliferation and differentiation (5).

We have previously reported the anti-proliferative effects of IP6 in the PANC-1 and MIAPACA pancreatic cancer cell lines, using identical physiologic IP6 doses (14). Herein, we reported profound reductions in cellular proliferation ranging from 37.1 to $91.5 \%$. We observed similar results when IP6 was administered to melanoma cells in vitro, with mean inhibitions of $52 \%$ after only $72 \mathrm{~h}$ of incubation (15). In our current study, significant growth inhibition was induced by the addition of IP6 in vitro to both Barrett's adenocarcinoma cell lines. These cell lines are useful in that one is wild-type p53 (SEG1) while the other is mutated (BIC-1). p53 mutation has been associated with Barrett's aggressiveness, and may be an early stage in the transformation of benign to malignant epithelium (26). This data in conjunction with the other studies cited above indicates that IP6 may have the potential to become an effective adjuvant treatment for Barrett's adenocarcinoma.

Among the reported mechanisms by which IP6 exerts its anti-proliferative effects are through regulation of angiogenesis and apoptosis. We previously evaluated the effects of in vitro IP6 administration on VEGF production in the HTB68 melanoma cell line (15). VEGF production was significantly reduced using IP6 by an astounding $81.7 \%$. These data indicate that IP6 acts as an anti-angiogenic agent, accounting for one of the possible mechanisms of action. We have also previously shown that IP6 increased early and late apoptotic activity in both PANC-1 and MIAPACA pancreatic cancer cell lines (14), as well as melanoma. In our current study, we observed increases in both early and late apoptotic activity in the SEG-1 cell line. Necrotic activity was also significantly 
increased. The observed increases in early apoptotic activity may indicate that the cells were in a static, non-proliferative state, while the increases in late apoptotic activity suggests that the cells were in the final stages of the apoptotic cycle and that cell death was imminent. The increase in necrosis observed in the SEG-1 cells indicates that a cytotoxic or cytostatic method of response was induced by IP6 treatment. Since apoptosis and necrosis were measured after only $18 \mathrm{~h}$ of incubation, it is possible that any effects of IP6 on these processes occurred later and were missed at this juncture in the BIC cell line, or be a function of its mutated p53 status.

The prevalence of IP6 compounds in safe, naturally occurring foods make it an attractive natural therapy for dietary and conventional treatment and possible prevention of esophageal cancer. Their effects in combination with conventional chemotherapy will be investigated. The results reported herein also encourage in vivo studies, and possibly lead to Phase II clinical trials in an effort to determine the clinical efficacy of these agents in the management or prevention of this disease.

Treatment of Barrett's adenocarcinoma in vitro with the common dietary polyphosphorylated carbohydrate IP6 decreased cellular growth by pro-apoptotic mechanisms. Our findings suggest that IP6 has the potential to become an effective adjunct for Barrett's adenocarcinoma. Further in vivo and human studies are needed to evaluate safety and clinical utility of this agent in patients with Barrett's adenocarcinoma.

\section{References}

1. Atherfold PA and Jankowski JA: Molecular biology of Barrett's cancer. Best Pract Res Clin Gastroenterol 20: 813-827, 2006.

2. Mehta S, Johnson IT and Rhodes M: Systematic review: the chemoprevention of oesphageal adenocarcinoma. Aliment Pharmacol Ther 22: 759-768, 2005

3. Buttar NS and Wang KK: Mechanisms of disease: carcinogenesis in Barrett's esophagus. Nat Clin Pract Gastroenterol Hepatol 1: 106-112, 2004.

4. Fox CH and Eberl M: Phytic acid (IP6), novel broad spectrum anti-neoplastic agent: a systematic review. Comp Ther Med 10: 229-234, 2002.

5. Vucenik I and Shamsuddin AM: Protection against cancer by dietary IP, and inositol. Nutr Cancer 55: 109-125, 2006.

6. Graf E and Eton JW: Antioxidant functions of phytic acid. Free Radical Biol Med 8: 61-69, 1990.

7. Shammsudin AM, Yang G-Y and Vucenik I: Novel anti-cancer functions of IP6: growth inhibition and differentiation of human mammary cancer cell lines in vitro. Anticancer Res 16: 3287-3292, 1996.
8. Shamsuddin AM and Vucenik I: Mammary tumor inhibition by IP6: a review. Anticancer Res 19: 3671-3674, 1999.

9. Jenab M and Thompson LU: Phytic acid in wheat bran affects colon morphology, cell differentiation and apoptosis. Carcinogenesis 21: 1547-1552, 2000.

10. Vucenik I, Zhang ZS and Shammsuddin AM: IP6 treatment of liver cancer II. Intra-tumoral injection of IP6 regresses preexisting human liver cancer xenotransplanted in nude mice. Anticancer Res 18: 4091-4096, 1998.

11. Shammsuddin AM and Yang GY: Inositol hexaphosphate inhibits growth and induces differentiation of PC-3 human prostate cancer cells. Carcinogenesis 16: 1975-1979, 1995.

12. Singh RP, Agarwal C and Agarwal R: Inositol hexaphosphate inhibits growth, and induces G1 arrest and apoptotic death of prostate carcinoma DU145: modulation of CDK1-CDK-cyclin and pRb-related protein-E2F complexes. Carcinogenesis 24: 555-563, 2003.

13. Agarwal C, Dhanalakshmi S, Singh RP and Agarwal R: Inositol hexaphosphate inhibits growth and induces G1 arrest and apoptotic death of androgen-dependent human prostate carcinoma LNCaP cells. Neoplasia 6: 646-659, 2004.

14. Somasundar P, Riggs DR, Jackson BJ and McFadden DW: Inhibition of melanoma growth by hemocyanin occurs via early apoptotic pathways. Am J Surg 190: 713-716, 2005.

15. Rizvi I, Riggs DR, Jackson BJ, Ng A, Cunningham $\mathrm{C}$ and McFadden DW: Inositol hexaphosphate (IP6) inhibits cellular proliferation in melanoma. J Surg Res 133: 3-6, 2006.

16. McFadden D, Riggs D, Jackson B and Davis L: In vitro effects of keyhole limpet hemocyanin in Barrett's adenocarcinoma of the esophagus. Am J Surg 186: 552-555, 2003.

17. Mosmann T: Rapid colorimetric assay for cellular growth and survival: application to proliferation and cytotoxicity assays. J Immunol Methods 65: 55-63, 1983.

18. Dixon WJ and Massey FJ: Introduction to Statistical Analysis. 4th edition. McGraw-Hill, New York, 1983.

19. Ludbrook J: Multiple comparison procedures updated. Clin Exp Pharmacol Physiol 25: 1032-1037, 1998.

20. De Meester TR: Barrett's esophagus. Surgery 113: 239-241, 1993.

21. Streitz JM Jr, Ellis FH Jr, Gibb SP, Balogh K and Watkins E Jr: Adenocarcinoma in Barrett's esophagus. A clinicopathologic study of 65 cases. Ann Surg 213: 122-125, 1991.

22. Jemal A, Siegel R, Ward E, Murray T, Xu J and Thun MJ: Cancer Statistics, 2007. CA Cancer J Clin 57: 43-66, 2007.

23. Devesa SS, Blot WJ and Fraumeni JF Jr: Changing patterns in the incidence of esophageal and gastric carcinoma in the United States. Cancer 83: 2049-2053, 1998.

24. Soldes OS, Kuick RD, Thompson IA, Hughes SJ, Orringer MB, Lannettoni MD, Hanash SM and Beer DG: Differential expression of Hsp27 in normal oesophagus, Barrett's metaplasia and oesophageal adenocarcinomas. Br J Cancer 79: 595-603, 1999.

25. Deliliers GL, Servida F, Fracchiolla NS, Ricci C, Borsotti C, Colombo G and Soligo D: Effect of inositol hexaphosphate [IP(6)] on human normal and leukaemic haematopoietic cells. Br J Haematol 117: 577-587, 2002.

26. Keswani RN, Noffsinger A, Waxman I and Bissonnette M: Clinical use of p53 in Barrett's esophagus. Cancer Epidemiol Biomarkers Prev 15: 1243-1249, 2006. 